\title{
In-House Diagnostic Testing opens up New Revenue Streams for PTs
}

\author{
Dimitrios Kostopoulos* \\ Clinical Affiliate Assistant Professor FAU, College of Medicine, USA
}

Submission: November 01, 2017; Published: November 21, 2017

*Corresponding author: Dimitrios Kostopoulos, ABPTS Board Certified in EMG/NCS Testing, Clinical Affiliate Assistant Professor FAU, College of Medicine, Hands On Diagnostics, USA, Tel: (917) 538-2242; Fax: (718) 732-2564; Email: dimi@handsonpt.org

\section{Editorial}

By training to deliver established diagnostic tests within the clinic, physical therapists are increasing net profit margins and improving patient outcomes.

In the traditional approach, physical therapists evaluate patients by conducting a physical therapy and orthopedic physical examination to derive the ideal treatment plan. When more sophisticated diagnostic tests are required, the patient is typically referred to a small universe of specialists in a process that can take several weeks to receive results.

Today, however, physical therapists are increasingly bringing this diagnostic testing into the clinic, investing in the equipment and training designed to reduce the learning curve and produce more immediate income. By doing so, clinics are able to expand the scope of their practice by tapping into a lucrative new universe of CPT codes while also deriving more effective treatment plans that improve patient outcomes.

"With declining reimbursements, we are always looking for new avenues in which to pursue greater revenue, particularly net profit margins," says Nathan Shields, co-owner of Rise Rehabilitation Specialists with 18 years as an orthopedic physical therapist. "We also want to differentiate our clinic by providing a service that is still within the physical therapy realm."

\section{Better diagnosis through objective testing}

Physical therapists have long sought more objective methods of diagnosis to supplement the normal physical evaluation.

"As physical therapists, a lot of what we do is relatively subjective testing," explains Shields. "Many of the tests are scientifically proven, but are still based upon the skill level and experience of the therapist."

In many cases, orthopedic evaluation tests may also miss pathologies and lead to ineffective treatment. More objective diagnostic tests allow physical therapists to troubleshoot issues with challenging patients quickly and accurately. This leads to better outcomes, with patients experiencing faster, more effective results.

"We are excited about the opportunity to diagnose patients with greater certainty. This is the only thing physical therapists can do to get some really objective diagnostic testing," adds Shields. Among the tests that physical therapists can be trained on include electromyography (EMG) nerve conduction testing, musculoskeletal and neuroultrasound, vestibular testing to treat patients with vertigo and balance problems and Evoked Potential tests.

\section{EMG testing}

EMG/NCS testing is one of the most reliable diagnostic tools for many dysfunctions of the Neuromuscular System. An EMG measures the electrical activity of muscles at rest and during contraction. Nerve Conduction Studies (NCS) measure the health of the nerves.

Measuring the electrical activity in muscles and nerves can help identify diseases that damage muscle tissue (such as myopathy, radiculopathy and muscular dystrophy) or nerves (such as amyotrophic lateral sclerosis or peripheral neuropathies). For symptoms such as numbness, weakness or tingling in an arm or leg, or conditions like sciatica and carpal tunnel syndrome, an EMG/NCS is a way to assess muscle and nerve function and is often used with other tests such as MRI and CT scan.

Physical therapists have been able to perform EMG and nerve conduction tests for more than 45 years. In 1978, the American Physical Therapy Association established a specialty program through the American Board of Physical Therapist Specialties for certification in clinical electrophysiology. Yet today there are 
only about 177 board certified physical therapists. This is due to the requirements for board certification, which are rigorous and include over 2000 direct clinical hours in the specialty area and completion of $500 \mathrm{EMG} / \mathrm{NCS}$ studies along with a vigorous written exam.

Most current educational models also do not offer the opportunity for revenue generation before the completion of the entire program which makes it challenging for the practicing therapist to commit to such program. EMG training can take between 1.5 to 3 years before the physical therapist can conduct the tests on patients. For most practicing physical therapist, this is not a workable model.

\section{Diagnostic testing training programs}
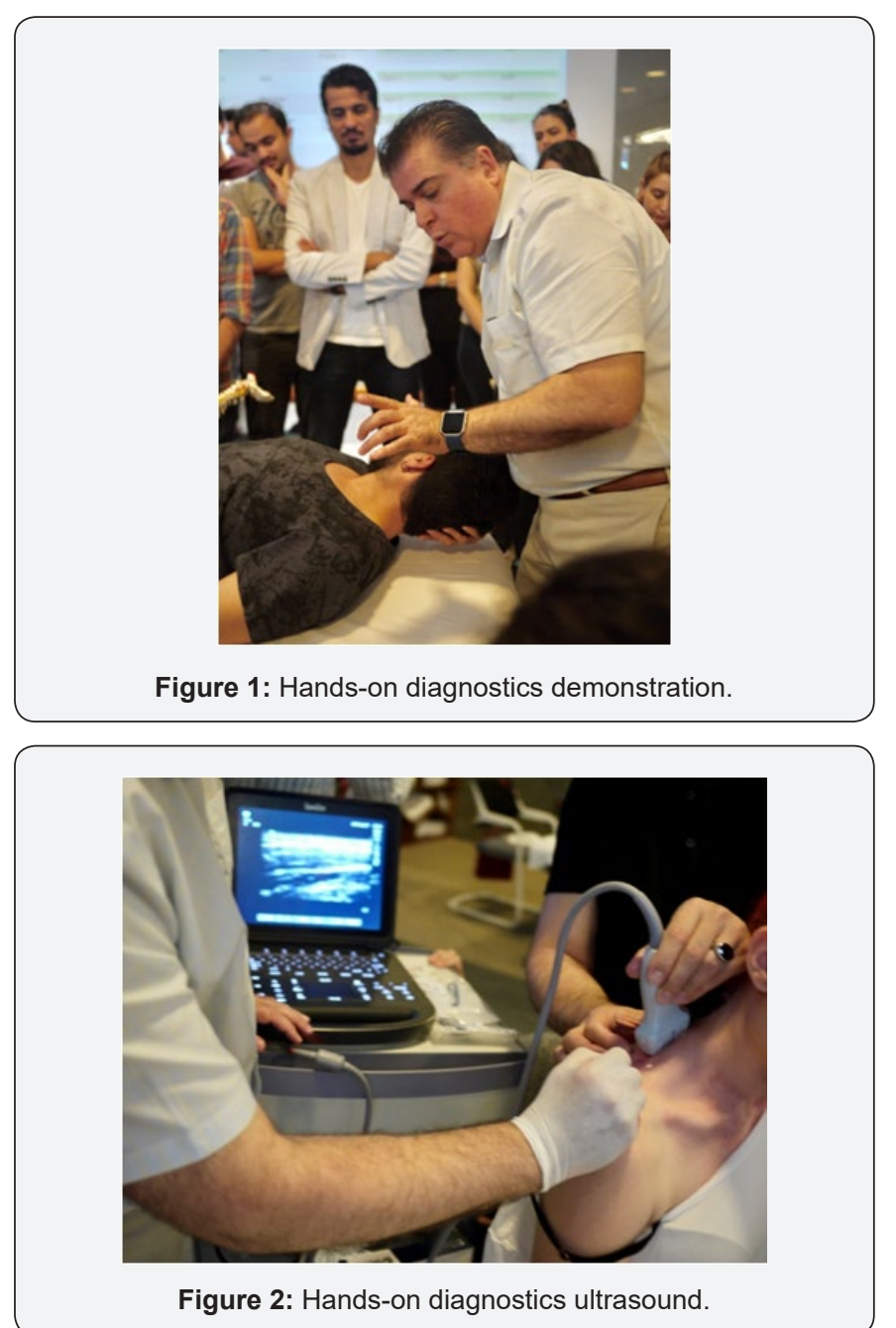

Today, training programs such as Hands-On Diagnostics (Figure 1\&2) exist to reduce the learning curve and speed the time to reimbursement. Hands-On Diagnostics is a national franchise organization that helps physical therapy practices to establish in-house diagnostic services for their patients. To date, the franchise has expanded to 16 different states with over 90 physical therapy facilities. The program is supported by live and virtual mentorships with board certified experts that allow the physical therapist to be able to perform these tests and get reimbursed almost immediately. In addition to training, the company provides marketing advice, promotional materials to provide to referring physicians, insight on how to approach other doctors and guidance on how to talk to patients about the tests, and how to bill and collect from insurance for this specialized type of service.

For the diagnostic equipment, the physical therapist must purchase it directly from the manufacturer. Shields first learned about Hands-On Diagnostics four years ago after his business partner Will Humphreys learned about the program from another therapist conducting the tests.

"It was an opportunity to add testing to what we were already doing," says Shields. "We could implement it pretty easily and quickly into our process to generate more revenue."

Of particular interest to Shields was the ability to offer musculoskeletal ultrasound. Ultrasound imaging uses sound waves to produce pictures of muscles, tendons, ligaments and joints throughout the body. It is used to help diagnose sprains, strains, tears, and other soft tissue conditions. Ultrasound is safe, noninvasive, and does not use ionizing radiation. According to Shields, his clinic was able to begin testing almost immediately. "After our first training session, we were able to perform the testing [with the supervision of the mentor]," says Shields. "As we committed ourselves to more and more testing, we experienced a significant increase in revenue." Perhaps more importantly, patients are experiencing better results. "We are now able to treat patients with more certainty and, in some cases, we have been able to use the testing to create protocols and get immediate responses on the physical therapy side," says Shields.

The ultimate goal of Hands-On Diagnostics is to provide a clear path for therapists to achieve board certification in clinical electrophysiology testing and certification in musculoskeletal ultrasound. Of the nine physical therapists certified in clinical electrophysiology in 2016, five were franchise members. Now that Hands-On Diagnostics has received approval as a residency developing program in clinical electrophysiology from the American Board of Physical Therapy Specialists, Shields says he intends to complete his residency and sit for the board certification examination less than 18 months from starting the program.

"As reimbursements decline, a practice either has to increase revenue through more aggressive marketing or finding other ways to make money," says Shields. "Diagnostic testing provides us a way to do that." 


\section{Your next submission with Juniper Publishers will reach you the below assets}

- Quality Editorial service

- Swift Peer Review

- Reprints availability

- E-prints Service

- Manuscript Podcast for convenient understanding

- Global attainment for your research

- Manuscript accessibility in different formats

( Pdf, E-pub, Full Text, Audio)

- Unceasing customer service

Track the below URL for one-step submission https://juniperpublishers.com/online-submission.php 